\title{
Doce lecciones para el desarrollo de indicadores locales de determinantes sociales de la salud
}

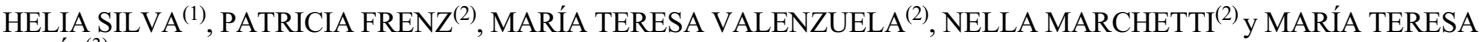
SOLÍS ${ }^{(3)}$

\section{INTRODUCCIÓN}

En el contexto de una nueva agenda social y tomando como base los Objetivos Sanitarios para la década 2000-2010, es que se acrecienta el compromiso en Chile por reducir las inequidades en salud.

Para apoyar a los equipos locales en esta tarea, a partir del año 2007 el Observatorio de Salud Pública (OSP) ha elaborado un instrumento con la finalidad de monitorear el avance de los determinantes sociales de la salud (DSS) estructurales e intermediarios a través del "Barómetro Comunal", que corresponde a un conjunto de indicadores agrupados en áreas y dimensiones, calculados con información disponible públicamente en la web.

Así, se pretende reflejar el estado actual de las temáticas relevantes para la equidad en salud, y permitir la comparación intercomunal, regional $\mathrm{y}$ nacional de sus valores, para en un futuro próximo evaluar sus tendencias y analizar asociaciones, de manera que constituya información relevante que apoye la toma de decisiones en políticas de salud a nivel local.

\section{LOS APRENDIZAJES PARA EL DESARROLLO DEL INSTRUMENTO}

Con la finalidad de compartir los aprendizajes con los diversos actores interesados en herramientas que apoyen el trabajo en DSS, el OSP presenta en este artículo doce lecciones que surgen del proceso de construcción y aplicación de los indicadores locales de equidad.

A continuación se detallan brevemente estos aprendizajes:

\section{Definir el modelo de observación: marco conceptual de determinantes sociales e inequidades en salud}

Concepto originado en la década de los años '70 pero con raíces históricas anteriores, actualmente los DSS se definen según la Organización Mundial de la Salud como las circunstancias en que las personas nacen, crecen, viven, trabajan y envejecen, y los sistemas establecidos para combatir las enfermedades. A su vez, esas circunstancias están configuradas por un conjunto más amplio de fuerzas: económicas, sociales, normativas y políticas ${ }^{1}$. Whitehead, los identifica como las condiciones sociales en las cuales viven y trabajan las personas $^{2}$, o simplemente, como lo señala Tarlov, son las características sociales dentro de las cuales la vida tiene lugar ${ }^{3}$.

Dada la preocupación por relevar la salud como un bien especial y la equidad como un imperativo moral sobre las cuales influyen e interactúan una multiplicidad de factores, es que la Comisión de DSS de la OMS construyó en el año 2005, un modelo sustentado en la justicia y equidad social, que satisface varios

(1) Becaria de Salud Pública. Facultad de Medicina. Universidad de Chile. Independencia 939. Santiago, Chile. helia_s@yahoo.com.

(2) Escuela de Salud Pública. Facultad de Medicina. Universidad de Chile e integrantes del equipo del Observatorio de Salud Pública. Santiago. Chile.

(3) Magíster Salud Pública. Universidad de Chile. 


\begin{tabular}{|c|c|}
\hline 12 & $\begin{array}{l}\text { Medir la efectividad del instrumento para la } \\
\text { toma de decisiones a nivel local }\end{array}$ \\
\hline 11 & $\begin{array}{l}\text { Fortalecer a los equipos locales para interpretar } \\
\text { y analizar sus indicadores }\end{array}$ \\
\hline 10 & $\begin{array}{c}\text { Avanzar en el análisis de tendencias y modelos } \\
\text { causales }\end{array}$ \\
\hline 9 & Reconocer las limitaciones del instrumento \\
\hline 8 & $\begin{array}{c}\text { Establecer referencias para la comparación: } \\
\text { semáforo }\end{array}$ \\
\hline 7 & $\begin{array}{c}\text { Calcular los indicadores y determinar su } \\
\text { distribución }\end{array}$ \\
\hline 6 & $\begin{array}{c}\text { Generar una base de datos para todas las } \\
\text { comunas del país }\end{array}$ \\
\hline 5 & $\begin{array}{l}\text { Traducir dimensiones conceptuales en } \\
\text { definiciones operativas de indicadores }\end{array}$ \\
\hline 4 & $\begin{array}{c}\text { Recopilar evidencia, experiencia e información } \\
\text { disponible }\end{array}$ \\
\hline 3 & $\begin{array}{l}\text { Construir el entendimiento común y } \\
\text { mecanismos de priorización }\end{array}$ \\
\hline 2 & Involucrar a los actores locales desde el inicio \\
\hline 1 & $\begin{array}{c}\text { Definir el modelo de observación: marco } \\
\text { conceptual de dss }\end{array}$ \\
\hline
\end{tabular}

Figura 1. Doce lecciones para el desarrollo de un modelo de indicadores locales de DSS.

requerimientos entre ellos: a) ofrece un marco conceptual respecto de cómo abordarlos, b) orienta sobre cómo los DSS producen inequidades en salud, c) sobre cómo se interrelacionan y d) permite identificar puntos de entrada para intervenciones, siempre con un marcado sello de intersectorialidad ${ }^{4-5}$. Esto constituyó la base para la definición de las dimensiones y áreas del modelo de observación de DSS locales que deben ser definidas operativamente en indicadores.

\section{Involucrar a los actores locales desde el inicio}

Teniendo en cuenta el objetivo de construir un instrumento útil para la comunidad y sus autoridades, es de vital importancia su participación continua desde el comienzo para tener una mirada real de aquellos puntos prioritarios relevantes al contexto.

Desde su inicio, participaron en la elaboración de la propuesta los profesionales del Departamento de Planificación y Desarrollo del Servicio de Salud Metropolitano Norte (SSMO) y directivos de salud de sus 9 comunas: La Reina, Las Condes, Lo Barnechea, Macul, Ñuñoa, Peñalolén, Providencia, Vitacura e Isla de Pascua; profesionales encargados de programas de salud de los Centros de Salud Familiar y autoridades locales, destacando particularmente la colaboración del equipo de Peñalolén.

Con todos ellos el Equipo del OSP guió el desarrollo de una propuesta inicial de indicadores, los que en diversas reuniones fueron analizados en cuanto a su pertinencia e importancia local.

\section{Construir el entendimiento común y un mecanismo de priorización}

El primer paso ${ }^{1}$ consistió en desarrollar una capacitación de todos los actores involucrados, con asistencia libre, ocasión en la que se examinó este nuevo enfoque del proceso saludenfermedad basado en el modelo de la OMS, con ejemplos atingentes de cómo abordarlos de modo holístico, con soluciones intersectoriales, que buscan y potencian la integralidad, transversalidad y participación comunitaria.

Asimismo, se trabajó con el equipo de Peñalolén una metodología de priorización de temas relevantes de observar en el espacio local, basada en modelos validados para la obtención de consenso (adaptación del método Delfos convencional, en tres rondas de consulta por correo electrónico), considerando que éste se debe producir entre un número considerable de personas (30), que se cuenta con tiempo limitado evitando traslados, y que se minimizan los costos humanos y financieros, de manera de hacer viable su ejecución.

Tras talleres, reuniones y discusiones, se hizo evidente la importancia de profundizar en un plan de capacitación, el cuál se ha ido estructurando gradualmente. 


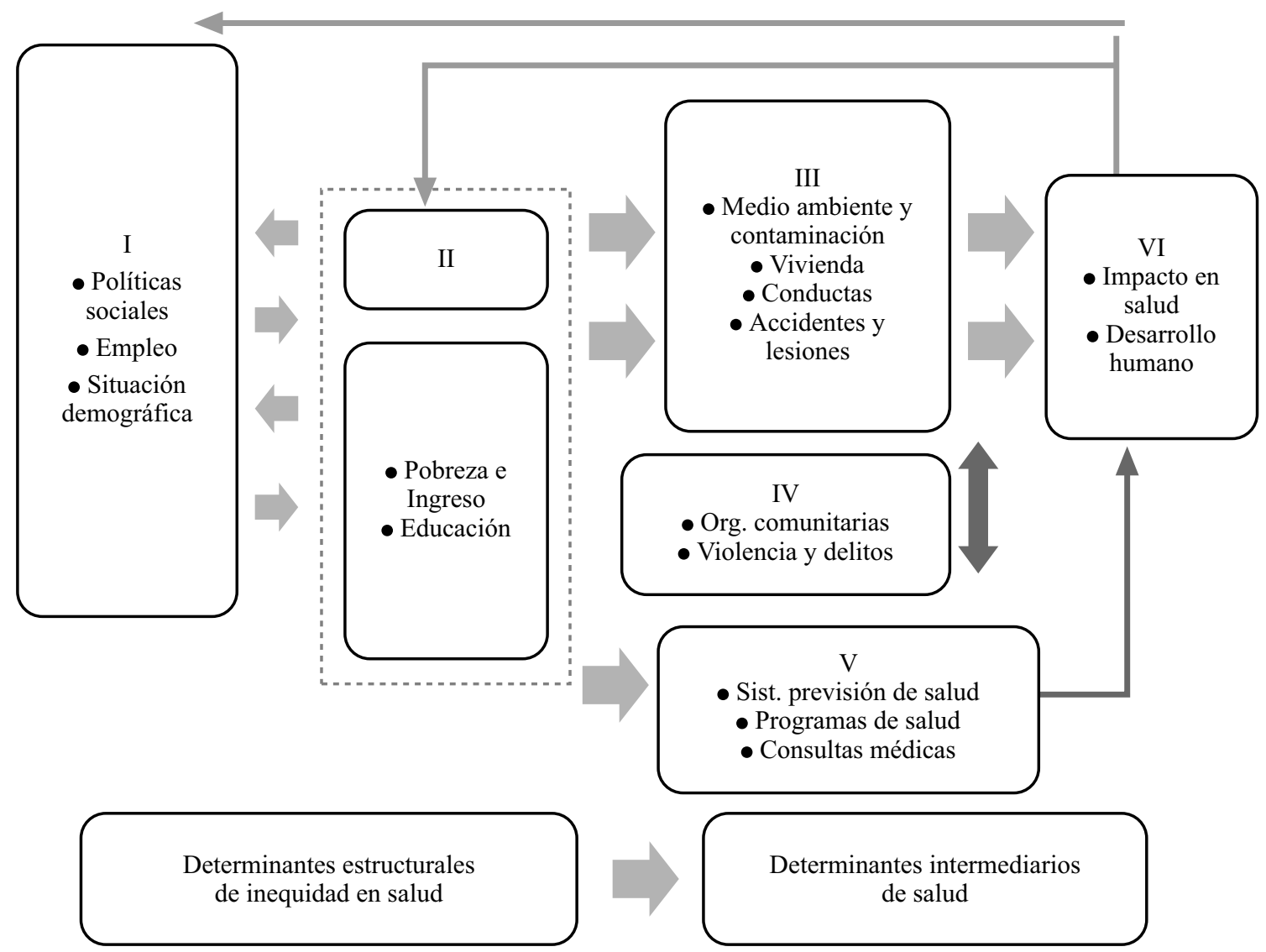

Figura 2. Dimensiones y áreas de los Indicadores para las comunas de Chile, clasificadas según el Modelo de DSS.

\section{Recopilar evidencia, experiencia e información disponible}

Inspirados en el trabajo realizado por el London Health Observatory (LHO), el equipo del OSP estableció etapas de trabajo para la selección y construcción de los indicadores:

a) Revisión de la canasta de 70 indicadores locales del LHO agrupados en las siguientes áreas ${ }^{6}$ :
- Materno-infantil
- Adulto mayor
- Grupos de riesgo
- Estilos de vida
- Educación
- Participación comunitaria

- Seguridad ciudadana

- Medio ambiente

- Empleo, pobreza y deprivación

- Acceso local a servicios

- Salud mental

- Accidentes

b) Cada área e indicador fueron analizados en cuanto a relevancia, disponibilidad y/o replanteamiento en el contexto nacional, en correspondencia razonable a las dimensiones relevantes del marco de los DSS, siempre teniendo en cuenta que la información estuviese disponible en la web para las 348 comunas de Chile, con el objeto de poder establecer comparaciones. Constituyeron fuentes de información: 
1. MINSAL: Ministerio de Salud: http://www.minsal.cl/

2. DEIS: Departamento de Estadísticas e Información de Salud, MINSAL: http://deis.minsal.cl/index.asp

3. MINVU: Ministerio de Vivienda y Urbanismo - Observatorio Urbano: http://www.observatoriourbano.cl

4. MIDEPLAN: Ministerio de Planificación Nacional, Base de datos de Encuesta de Caracterización Socioeconómica Nacional (CASEN) 2006:

http://www.mideplan.cl/casen/ y CASEN interactiva:

http://celade.cepal.org/redatam/paises/chl/mi deplanii/casen2006/index.html

5. MIDEPLAN: Unidad Sistema Integrado de Información Social: http://siis.mideplan.cl/indicador/

6. PNUD: Programa de las Naciones Unidas para el Desarrollo: http://www.pnud.cl/

7. MINVU Observatorio Habitacional, http://www.observatoriohabitacional.cl

8. MINEDUC: Ministerio de Educación: www.mineduc.cl

9. JUNAEB: Junta Nacional de Auxilio Escolar y Becas: www.junaeb.cl

10. MINISTERIO DEL INTERIOR, Sistema Nacional de información Municipal: http://www.sinim.gov.cl/ficha_comunal

11.MINISTERIO DEL INTERIOR, Subsecretaria de Desarrollo Regional y Administrativo: www.subdere.gov.cl

12.MINISTERIO DEL INTERIOR, www.seguridadciudadana.gob.cl

13. MINISTERIO DE TRANSPORTES Y TELECOMUNICACIONES, Comisión Nacional de Seguridad de Tránsito: http://www.conaset.cl

\section{Traducir las dimensiones conceptuales en operativas: propuesta final de indicadores}

El proceso de definición de los indicadores no estuvo ausente de dificultades, tales como comenzar con información deseable que se redujo a la posible, siendo ésta la que es registrada sólo para algunas comunas, para finalmente terminar con lo factible, que es aquella disponible para todas las comunas del país.

Dada la complejidad per se que ofrecen las dimensiones, se estimó importante explicitar la traducción a definiciones operativas siendo éstas los indicadores, a través de una ficha explicativa que precisa la definición, justificación de su incorporación, utilidad local, fórmula o método de cálculo, fuente de información, periodicidad y año más reciente de obtención, posibilidades de desagregación como por ejemplo: sexo, grupo social o territorial y, por último, comentarios y limitaciones cuando son atingentes.

Tomando ésta base, los indicadores fueron categorizados según si correspondían a determinantes estructurales o intermediarias y, posteriormente, agregadas en seis dimensiones, cada una compuesta de áreas y éstas últimas de indicadores como se muestra a continuación:

Estadísticos descriptivos

\begin{tabular}{|c|c|c|c|c|c|}
\hline & $\mathbf{N}$ & Mínimo & Máximo & Media & D.S. \\
\hline$\%$ & 325 & 0,61 & 28,23 & 12,187 & 5,956 \\
\hline $\begin{array}{c}\mathbf{N} \\
\text { válido }\end{array}$ & 325 & Vitacura & Lebu & & \\
\hline
\end{tabular}

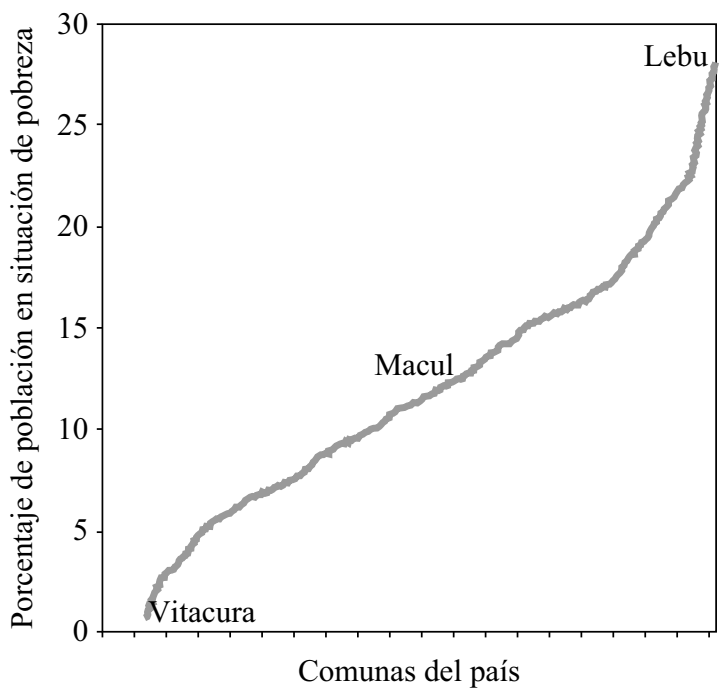

Figura 3. Situación nacional para el porcentaje de población en situación de pobreza. 
Estadísticos descriptivos

\begin{tabular}{|c|c|c|c|c|c|}
\hline & $\mathbf{N}$ & Mínimo & Máximo & Media & D.S. \\
\hline$\%$ & 7 & 0,61 & 12,68 & 5,12 & 4,02 \\
\hline $\begin{array}{c}\mathbf{N} \\
\text { válido }\end{array}$ & 7 & Vitacura & Macul & & \\
\hline
\end{tabular}

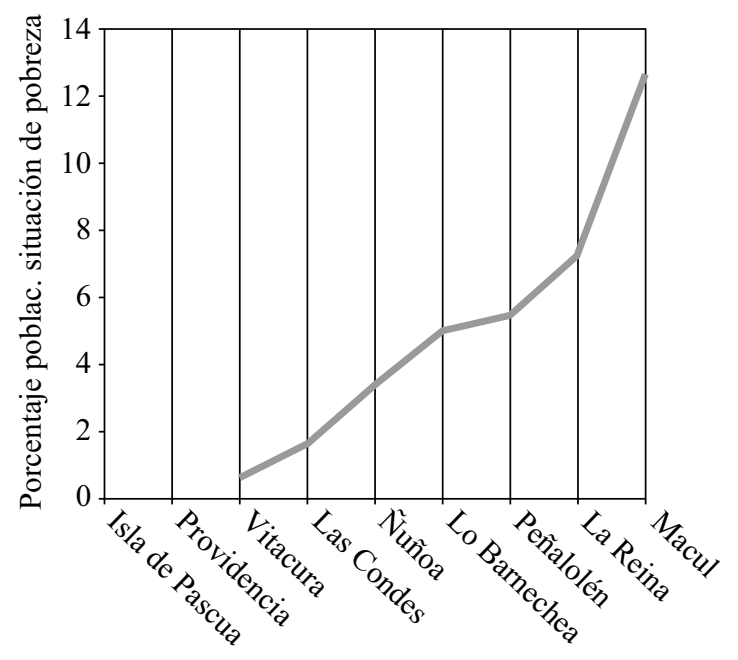

Comunas del SSM-Oriente

Figura 4. Situación de las comunas del SSMO para el porcentaje de población en situación de pobreza.

\section{Contexto social, económico, político y demográfico}

\section{Área políticas-sociales}

- Gasto total municipal por habitante

- Índice de financiamiento municipal

\section{Área empleo}

- Tasa de desocupación total, masculina, femenina y en menores de 20 años.

- Tasa de participación laboral total, masculina y femenina.

- Porcentaje de trabajadores independientes

\section{Área situación demográfica}

- Porcentaje de población de 0 a 14 años, de 15 a 64 años y mayor de 65 años.

- Índice de Vejez

- Índice de Dependencia

\section{Posición socio-económica}

\section{Área pobreza e ingresos}

- Porcentaje de población en situación de pobreza e indigencia

- Porcentaje de hogares pobres con jefatura femenina.

- Porcentaje de población beneficiaria de Bono Chile Solidario.

\section{Área educación}

- Puntajes P.S.U. en establecimientos municipalizados y particularessubvencionados.

- Puntaje SIMCE en $4^{\mathrm{a}}$ año de educación básica en matemáticas y lenguaje.

\section{Circunstancias materiales y conductas}

\section{Área medio ambiente y contaminación}

- Porcentaje de habitantes que percibe carencia de áreas verdes

- Tasa de motorización

\section{Área vivienda}

- Porcentaje de hogares en situación de hacinamiento

\section{Área conducta}

- Porcentaje de lactantes menores con lactancia materna exclusiva a los 6 meses.

\section{Área accidentes y lesiones}

- Tasa de siniestralidad

- Porcentaje de accidentes de tránsito, asociados a la ingesta alcohólica.

- Tasa de lesionados por accidente de tránsito.

- Porcentaje de lesionados por accidentes de tránsito, asociados a la ingesta alcohólica.

- Tasa de mortalidad por accidentes de tránsito.

- Porcentaje de fallecidos por accidentes de tránsito, asociados a la ingesta alcohólica. 


\section{Cohesión social}

Área organizaciones comunitarias

Tasa de organizaciones comunitarias

\section{Área violencia y delitos}

- Tasa de denuncias por delito de violencia intrafamiliar

- Tasa de denuncias por delitos de mayor connotación social

\section{Sistemas de salud}

Área sistemas de previsión de salud

- Porcentaje de inscritos en Fonasa, Isapre $\mathrm{y}$ en otro sistema.

\section{Área programas de salud}

- Porcentaje de nacidos vivos de madres menores de 15 años.

- Porcentaje de adolescentes entre las embarazadas en control.

- Cobertura efectiva de Hipertensión Arterial y Diabetes Mellitus.

\section{Área consultas}

- Número de consultas médicas totales, per capita anuales

\section{Impacto en equidad en salud y bienestar}

Área impacto en salud

- Tasa de mortalidad infantil

- Porcentaje de nacidos vivos con bajo peso

- Tasa de mortalidad específica por enfermedades cardiovasculares y por cáncer, entre 20 y 64 años.

- Prevalencia de malnutrición por déficit y exceso en menores de 6 años bajo control.

\section{Área desarrollo humano}

- Valor del IDH global, y de sus dimensiones: salud, educación, ingresos y variación entre 1994-2003.

\section{Generar una base de datos}

Desde las fuentes de información señaladas se recopilaron datos cuya calidad presentó algunos inconvenientes, tales como ausencia del registro del valor para ciertos indicadores, tipificación del valor no consignado como guión, casilla vacía o cero, siendo este último no diferenciable del cero registrado como sin eventos, construyendo así los indicadores. Se utilizaron los programas EXCEL, STATTRANSFER, STATA 10 y SPSS 11.5. para elaborar la base de datos.

Los valores calificados como outliers fueron eliminados por desviar las medidas resumen obtenidas tanto a nivel nacional como comunal.

Se destaca que la mayoría de los datos ausentes en el registro de valores de indicadores, se concentran en las comunas rurales de pocos habitantes.

\section{Calcular los indicadores y determinar su distribución}

Se elaboró estadísticas descriptivas para todos los indicadores seleccionados y se graficó su distribución en dos representaciones en SPSS, que ubican los valores de menor a mayor a lo largo de las comunas, una nacional, y otra para el SSMO. De esta forma, permite ubicar la situación de cada comuna en el contexto país o dentro del servicio de salud respectivo. Para ambos casos se adjuntó una pequeña tabla que indica el número de datos válidos considerados en la realización del gráfico, el valor mayor y menor obtenido, la media aritmética y una desviación estándar.

En la página web del OSP, se encontrará la guía que orientará la construcción de gráficos dados los datos disponibles en caso de querer realizarse para otros servicios de salud o a nivel regional ${ }^{7}$.

Próximamente, estos gráficos estarán disponibles en formato interactivo, así al posicionarse sobre cualquier punto de la curva aparecerá la comuna que aportó con tal valor.

\section{Establecer referencias para la comparación: semáforo}

El semáforo constituye un componente más de este instrumento llamado "Barómetro Comunal" y corresponde a una representación resumida de los indicadores, en que se califica el valor comunal respecto de su ubicación en el 


\begin{tabular}{|c|c|c|c|c|c|}
\hline Indicador & $\begin{array}{l}\text { Prom } \\
\text { País }\end{array}$ & $\begin{array}{l}\text { Mejor } \\
\text { SSMO }\end{array}$ & $\begin{array}{l}\text { Peor } \\
\text { SSMO }\end{array}$ & $\begin{array}{l}\text { Prom } \\
\text { SSMO }\end{array}$ & SSMO \\
\hline $\begin{array}{c}\text { Tasa de mortalidad infantil } \\
\text { *cada } 1000 \text { RNV }\end{array}$ & 8,65 & $\begin{array}{c}0,00 \\
\text { Isla de Pascua }\end{array}$ & $\begin{array}{l}9,80 \\
\text { Providencia }\end{array}$ & 6,18 & \\
\hline \% nac. vivos con bajo peso & 5,55 & $\begin{array}{l}5,40 \\
\text { Macul }\end{array}$ & $\begin{array}{c}11,70 \\
\text { Isla de Pascua }\end{array}$ & 6,96 & \\
\hline $\begin{array}{c}\text { Tasa de mort espec. por enf. cardiovascular } \\
20-64 \text { años } \\
* \text { cada } 100.000\end{array}$ & 53,68 & $\begin{array}{c}16,80 \\
\text { Lo Barnechea }\end{array}$ & $\begin{array}{c}157,90 \\
\text { Isla de Pascua }\end{array}$ & 46,20 & \\
\hline $\begin{array}{l}\text { Tasa de mort. Espec .por cáncer } \\
20-64 \text { años } \\
\text { *cada } 100.000\end{array}$ & 68,43 & $\begin{array}{c}46,60 \\
\text { Lo Barnechea }\end{array}$ & $\begin{array}{c}78,90 \\
\text { Isla de Pascua }\end{array}$ & 66,49 & \\
\hline $\begin{array}{c}\text { Prevalencia malnutrición por déficit } \\
* \text { cada } 100\end{array}$ & 6,24 & $\begin{array}{c}4,50 \\
\text { Isla de Pascua }\end{array}$ & $\begin{array}{l}7,70 \\
\text { Providencia }\end{array}$ & 6,40 & \\
\hline $\begin{array}{c}\text { Prevalencia malnutrición por exceso } \\
\text { * cada } 100\end{array}$ & 25,11 & $\begin{array}{c}21,10 \\
\text { Las Condes }\end{array}$ & $\begin{array}{l}27,80 \\
\text { La Reina }\end{array}$ & 24,34 & \\
\hline
\end{tabular}

Figura 5. Comparación de indicadores de impacto en salud para las comunas del SSMO y su situación en relación al promedio nacional (semáforo).

rango nacional (rojo - tercio inferior, amarrillo - tercio medio, y verde - tercio superior), lo que permite la comparación intercomunal salvaguardando las disquisiciones que ofrece su construcción y las limitaciones de su interpretación, que impactan negativamente su valor como por ejemplo: tasa de mortalidad infantil elevada en las comunas del SSMO en donde se emplazan recintos hospitalarios pediátricos.

\section{Reconocer las limitaciones del instrumento}

Entre las principales limitaciones se destacan posibles falencias técnicas en la falta de definiciones rigurosas y de criterios utilizados para precisar indicadores, especialmente por restricciones en el acceso a datos públicos de todas las comunas, subnotificación y mal registro de datos, errores de interpretación del valor cero los cuales alteran la sistematización de la información; no ponderación y pareamiento de datos, y finalmente la carencia de recursos humanos y financieros para profundizar en el análisis.

Las principales restricciones tienen que ver con la minería de datos para la construcción de las bases de datos de indicadores. Por eso el equipo del OSP valora la iniciativa del Ministerio de Salud de recopilar y difundir los indicadores de determinantes sociales y equidad para las regiones, publicados como Fichas Regionales del Diagnóstico de Salud ${ }^{8}$. Actualmente, trabajan en un instrumento comunal. Es de esperar que el acceso público a estas bases de datos constituya una fuente de información para quienes se interesen por seguir trabajando en esta línea.

\section{Avanzar en el análisis de tendencias y modelos causales}

Una vez terminado y afinado el proceso descriptivo, el OSP seguirá con la etapa analítica para establecer modelos explicativos de los principales determinantes sociales asociados, con diferentes resultados de salud a nivel comunal, ponderados respecto a su importancia relativa local.

Con la participación activa de los diferentes actores involucrados en la toma de decisiones y la obtención de datos aportados por nuevas encuestas y otras fuentes de información, se espera actualizar y complementar la base de 
datos y posteriormente analizar sus tendencias.

En lo inmediato, se pretende realizar un cruce de indicadores trazadores de cada dimensión, con el objetivo de determinar el grado de asociación entre éstos.

\section{Fortalecer la capacidad de los equipos locales para analizar e interpretar sus indicadores}

A pesar de las limitaciones del presente instrumento se estima que es un aporte porque cuenta con el trabajo de los diversos agentes involucrados en el proceso salud-enfermedad, muestra una representación esquemática de la posición comunal y nacional con la finalidad de conocer su situación actual y, por último, entrega orientaciones de cómo facilitar la construcción de un modelo de trabajo en DSS, a través de la consecución de etapas y focalización de recursos humanos, técnicos y financieros en las intervenciones deseadas a través de un trabajo colaborativo e intersectorial.

El equipo tiene consciencia que este tipo de herramienta debe ser aprehendida por los equipos locales, y para ello deben contar con el conocimiento, capacidades y recursos para su desarrollo y aplicación.

\section{Medir la efectividad del instrumento para la toma de decisiones a nivel local}

La finalidad de esta construcción es permitir a cada comuna identificar áreas críticas para informar y sustentar su accionar y, a su vez, permitir medir el impacto de diversas políticas y programas sociales tanto de salud como de otros sectores relativos al bienestar de la población.
La prueba final es su utilidad y uso en la toma de decisiones de los equipos locales, aspecto que debe ser evaluado.

\section{REFERENCIAS}

1. CDSS. Subsanar las desigualdades en una generación. Comisión sobre Determinantes Sociales de la Salud. OMS. Ginebra 2008. Disponible en: http:/www.who.int/social determinants/thecommis sion/finalreport/key_concepts/es/index.html. Consultado el 02/01/2010

2. WHITEHEAD M. The concepts and priniciples of equity and health. International Journal of Health Services. 1992; 22(3):429-445

3. TARLOV A, Social determinants of Health: the sociobiological transition, Blane D, Brunner E, Wilkinson D (eds), Health and Social Organization. London. Routledge. Pp. 71-93.

4. VEGA J, SOLAR O, IRWIN A, HERNÁNDEZ S, SANDOVAL H, DELGADO I, ET AL. En La perspectiva de la equidad, determinantes sociales de la salud en Chile. Equidad y determinantes sociales de la salud 2005; 9-18.

5. FRENZ P. Desafíos en salud pública de la Reforma: equidad y determinantes sociales de la salud. Rev. Chi. Salud Pública 2005: Vol. 9(2) 103-109.

6. LHO Local basket of Indicators. London Health Observatory. London. Disponible en: http://www.lho.org.uk/LHO_Topics/Analytic_Tools /BasketOfIndicatorsDataTool.aspx. Consultado el 02/01/2010

7. OSP. Barómetro Comunal. Observatorio de Salud Pública. Escuela de Salud Pública. Facultad de Medicina. Universidad de Chile. www.observatoriosaludpublica.cl

8. MINSAL. Fichas Regionales. www.equidad.cl/fichas/index.html

Recepción: 26 de marzo de 2010 Aprobación: 8 de abril de 2010

Usted puede comentar éste y otros artículos publicados en la Revista Chilena de Salud Pública, enviando un correo electrónico a revistasp@med.uchile.cl 